LUC DURRIEU DE MADRON ${ }^{1}$

\title{
Estimation de l'impact de différents modes d'exploitation forestière sur les stocks de carbone en Afrique centrale
}

Sébastien BAUWENS

Adeline GIRAUD ${ }^{1}$

Didier HUBERT ${ }^{3}$

Alain BILLAND 2

${ }^{1}$ Onfi

2, avenue de Saint-Mandé

75012 Paris

France

2 Cirad

Ur B\&sef

Campus international de Baillarguet

34398 Montpellier Cedex 5

France

3 Cirad

Projet Parpaf

Bangui

République centrafricaine

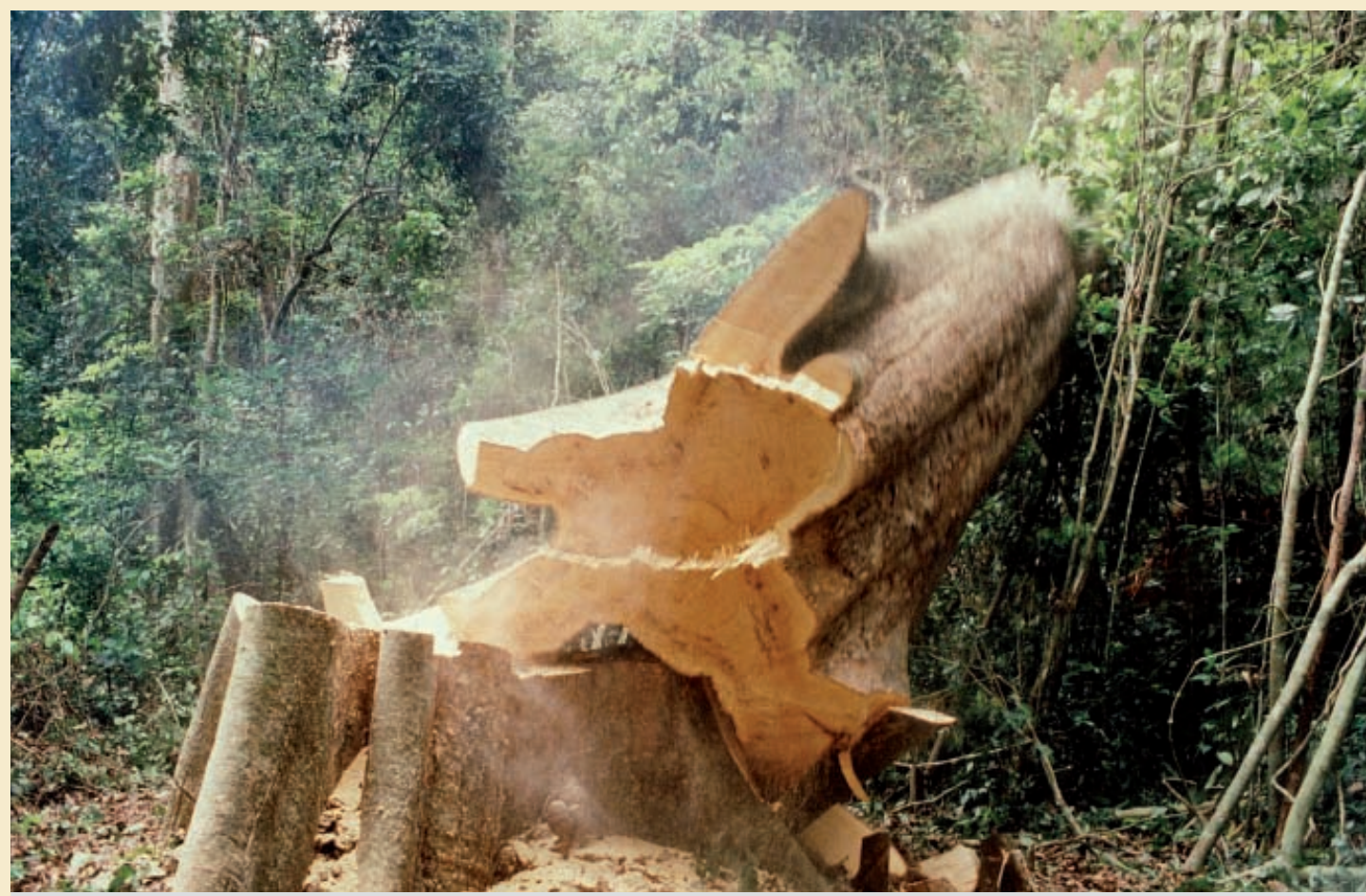

Abattage d'ayous, Triplochiton scleroxylon, en République centrafricaine. Photo L. Durrieu de Madron. 


\section{RÉSUMÉ}

\section{ESTIMATION DE L'IMPACT DE DIFFÉRENTS MODES D’EXPLOITATION FORESTIĖRE SUR LES STOCKS DE CARBONE EN AFRIQUE CENTRALE}

\section{ABSTRACT}

\section{ASSESSING THE IMPACT OF DIFFERENT LOGGING METHODS ON CARBON STOCKS IN CENTRAL AFRICA}

L'impact, en termes d'émission de carbone, d'une exploitation forestière n'a fait l'objet que de rares et réels travaux de recherche en Afrique centrale. Pourtant, dans le cadre des mécanismes Redd (Réduction des émissions dues à la déforestation et à la dégradation des forêts), cette problématique comporte des enjeux importants avec l'augmentation du nombre de concessions aménagées et l'importance grandissante des techniques d'exploitation à faible impact. Cette étude constitue un essai de méta-analyse incluant les diverses expériences issues de la littérature. Les données ainsi récoltées ont été appliquées à une exploitation hypothétique, afin de dresser un bilan théorique de l'impact sur le stock de carbone. Les résultats montrent qu'avec les hypothèses retenues l'aménagement d'une forêt permettrait une réduction du dixième des pertes en carbone, pour un prélèvement à l'hectare de 10 à $20 \mathrm{~m}^{3}$ de bois d'œuvre; et cela par rapport à une concession forestière conventionnelle sans gestion prévisionnelle. L'essentiel de l'économie en carbone proviendrait de l'augmentation des diamètres minimums d'exploitation. Par ailleurs, les gains que pourrait induire la pratique d'une exploitation à faible impact seraient ceux découlant de l'amélioration du tracé des pistes et des routes, à savoir 5 à $8 \%$ des pertes en carbone dues à l'exploitation conventionnelle. Ces gains sont supérieurs à l'erreur constatée sur la mesure de biomasse par coupe rase, la plus proche de la réalité, faite en Guyane. Néanmoins, ces gains sont largement inférieurs aux erreurs calculées sur l'estimation de la biomasse pour plusieurs études disponibles dans la littérature. Quoi qu'il en soit, la mise sous aménagement d'une concession forestière et l'utilisation de techniques d'exploitation à faible impact sont à même d'entraîner une différence favorable du stock de carbone en forêt dense africaine.

Mots-clés : exploitation à faible impact, aménagement, biomasse, Afrique.
There has been little in-depth research to date on logging impacts in Central Africa in terms of carbon emissions. The REDD mechanism (Reducing Emissions from Deforestation and forest Degradation), has brought this topic to the forefront, with the increase in managed logging concessions and the spread of low-impact logging methods. The aim of this study was to make a meta-analysis covering different experiences reported in the literature. The data collected were applied to a hypothetical logging operation in order to produce a theoretical assessment of its impact on carbon stocks. Our results show that, based on the assumptions used, managed forestry in a conventional logging concession with no forward planning would reduce carbon losses by one tenth, with 10 to $20 \mathrm{~m}^{3}$ of timber extracted per hectare. Most of the carbon savings would result from an increase in the minimum logging diameter. The other gains from low-impact logging would result from improving the design of logging roads, i.e., 5 to $8 \%$ of carbon losses due to conventional logging practice. These gains are higher than the error found for the nearest reality measurements made for clear-felled biomass in French Guiana. However, they are much lower than the errors calculated for biomass estimations in several studies available in the literature. In any case, introducing development planning and low-impact logging techniques in timber concessions are able to induce a favourable difference to carbon stocks in Africa's dense forests.

Keywords: low-impact logging, managed forestry, biomass, Africa.

\section{RESUMEN}

\section{ESTIMACIÓN DEL IMPACTO DE DISTINTOS MODOS DE APROVECHAMIENTO FORESTAL EN LAS RESERVAS DE CARBONO DE ÁFRICA CENTRAL}

El impacto de una explotación forestal en términos de emisión de carbono ha sido raramente contemplado en trabajos de investigación reales en África Central. Sin embargo, en el marco de los mecanismos REDD (Reducción de Emisiones por la Deforestación y la Degradación), este problema plantea importantes desafíos con el incremento del número de concesiones sujetas a ordenación y la creciente importancia de las técnicas de aprovechamiento de bajo impacto. Este estudio constituye un ensayo de metanálisis que incluye las distintas experiencias procedentes de la literatura existente. Los datos así obtenidos se aplicaron a una hipotética explotación para elaborar un balance teórico del impacto en las reservas de carbono. Los resultados ponen de manifiesto que, con las hipótesis adoptadas, la ordenación de un bosque permitiría una reducción de la décima parte de las pérdidas de carbono, para una extracción por hectárea de 10 a $20 \mathrm{~m}^{3}$ de madera de construcción; todo ello comparado con una concesión forestal convencional sin manejo planificado. La parte fundamental en el ahorro del carbono procedería sobre todo del aumento de los diámetros mínimos de aprovechamiento. Por otra parte, los beneficios que podrían impulsar la práctica de un aprovechamiento de bajo impacto serían los derivados de la mejora del trazado de pistas y carreteras, esto es: del 5 al $8 \%$ de las pérdidas de carbono debidas al aprovechamiento convencional. Estos beneficios son superiores al error constatado en la medición de biomasa por tala total, la más cerca de la realidad, hecho en Guayana francesa. Sin embargo, dichos beneficios son muy inferiores a los errores calculados en la estimación de la biomasa en varios estudios disponibles en la literatura científica. En cualquier caso, la implementación de la ordenación en una concesión forestal y el uso de técnicas de aprovechamiento de bajo impacto son capaces de generar una diferencia favorable de las reservas de carbono en el bosque denso.

Palabras clave: aprovechamiento de bajo impacto, ordenación, biomasa, África. 


\section{Introduction}

À l'échelle mondiale, parmi les émissions annuelles de gaz à effet de serre générées par les activités humaines depuis 1990, 15 à 25 pour cent (\%) proviennent du changement d'usage des sols, avec comme principal facteur la déforestation en zone tropicale (DENMAN, BRASSEUR, 2007). À cette déforestation émettrice de gaz carbonique $\left(\mathrm{CO}_{2}\right)$ se rajoute la dégradation des forêts tropicales qui peut produire des émissions de $\mathrm{CO}_{2}$ importantes. Ainsi, en Amazonie, chaque année, 27 à 50 millions de mètres cubes $\left(\mathrm{m}^{3}\right)$ de bois sont extraits, libérant environ 100 millions de tonnes (t) de carbone dans l'atmosphère (AsNer, 2006).

En Afrique centrale, l'impact des prélèvements de bois commercial contribuant à cette dégradation n'est guère connu, alors que $30 \%$ de la superficie forestière est parcourue par l'exploitation du bois d'œuvre (LAPORTE et al., 2007). Au sein des concessions de cette région, sont pratiqués différents modes d'intervention qui vont de l'exploitation conventionnelle à l'aménagement durable suivant des techniques d'" exploitation forestière à faible impact ». Pourtant, des études comparatives de l'impact sur les stocks de carbone de ces différents modes d'exploitation font défaut dans la région. Elles permettraient d'évaluer le gain en carbone issu de l'amélioration des techniques d'exploitation ainsi que de déterminer si les prélèvements les moins destructeurs sont à même de limiter la réduction du stock de carbone et de favoriser sa reconstitution (phénomène de résilience). Ces données et informations s'avèrent essentielles pour l'actuel mécanisme Redd (Reduced Emissions from Deforestation and Forest Degradation) qui cherche à rémunérer l'évitement des émissions de $\mathrm{CO}_{2}$ dues à la déforestation et à la dégradation des forêts.

L'étude propose une première approche pour estimer l'impact sur les stocks de carbone en forêt tropicale humide, en identifiant d'abord les pratiques d'exploitation susceptibles d'influencer les stocks de carbone dans les concessions forestières d'Afrique centrale ; sous l'angle d'une exploitation forestière industrielle et dans un cadre légal du respect des diamètres minimums exploitables (Dme). Ensuite, les impacts potentiels sur les stocks de carbone de différents modes d'exploitation ont été comparés à

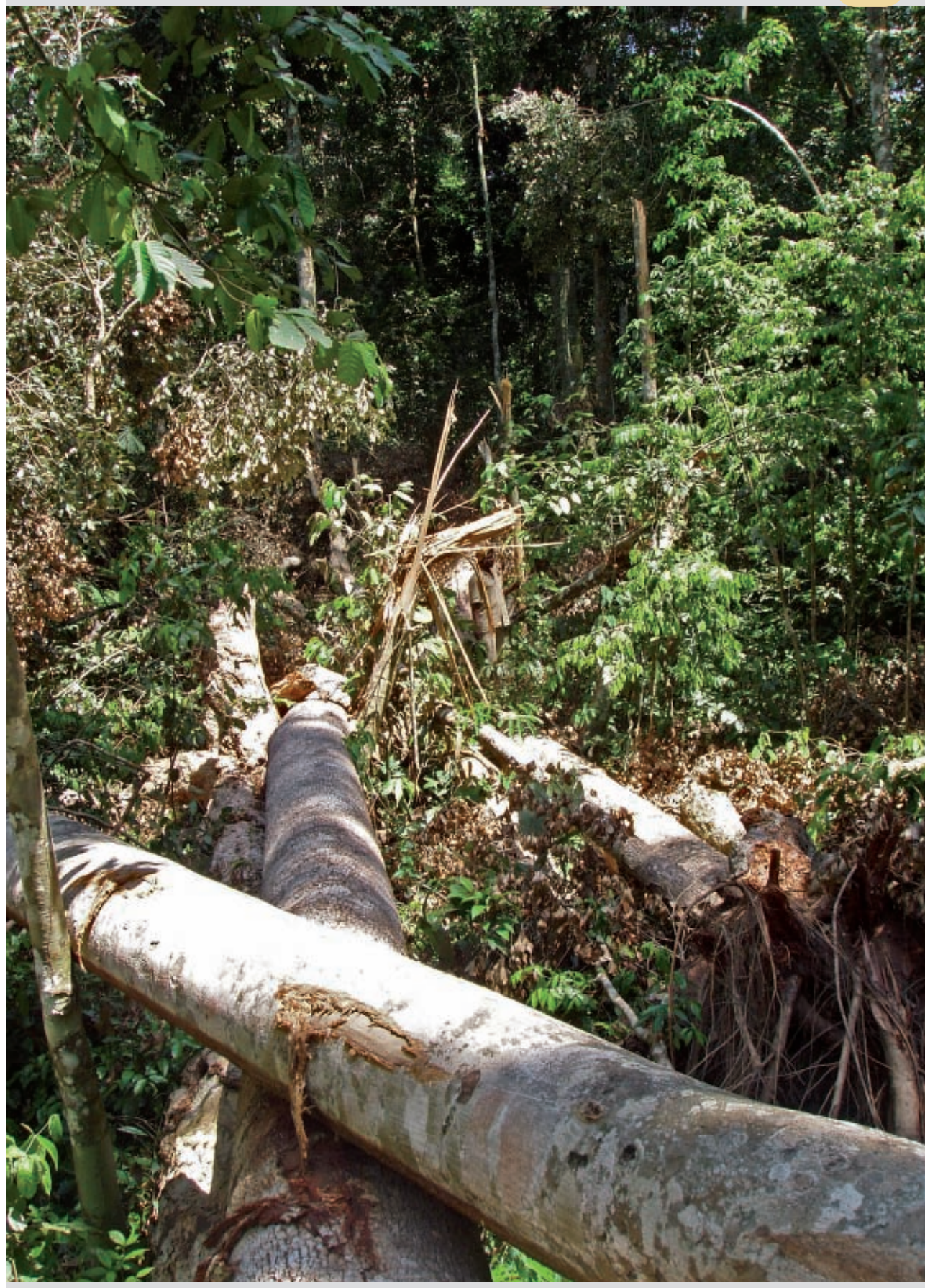

Dégâts d'abattage en République centrafricaine.

Photo L. Durrieu de Madron. partir des données d'aménagement issues de plusieurs concessions du bassin du Congo et de celles puisées dans la littérature. Les résultats ainsi obtenus ont permis de dégager les grandes tendances.

Le devenir du carbone n'a pas été considéré car, lors de l'exploitation, une partie du carbone reste dans l'écosystème. De l'arbre sur pied, il se retrouve dans la litière, dans les horizons humifères du sol, sous forme de bois en cours de décomposition ou stocké sous forme de bois d'œuvre. 


\section{Matériel et méthode}

\author{
Les modes d'exploitation forestière
}

Il est possible de retenir trois grandes catégories d'exploitation forestière en Afrique centrale :

- l'exploitation traditionnelle ou conventionnelle, sans aménagement $(\mathrm{Ec})$;

- l'exploitation sous plan d'aménagement (Espa) ;

- l'exploitation sous plan d'aménagement, faisant en plus appel aux techniques d'exploitation forestière à faible impact (Efi).

Pour le déroulement cohérent de l'étude, celle-ci se place dans le cas d'une exploitation industrielle (non artisanale) et d'un cadre légal dans lequel les diamètres minimums d'exploitabilité administratifs (Dme) sont respectés. En fait, sur le terrain, les catégories d'exploitation ne sont pas aussi tranchées ; il s'agit davantage d'un continuum entre les entreprises les plus « vertueuses » pratiquant des techniques d'exploitation à faible impact et les entreprises se situant à la limite de la légalité avec des pratiques peu durables. Par ailleurs, les pratiques, au sein d'une même concession forestière, peuvent varier d'un secteur à un autre en fonction des caractéristiques de terrain ou de la structure de la forêt.

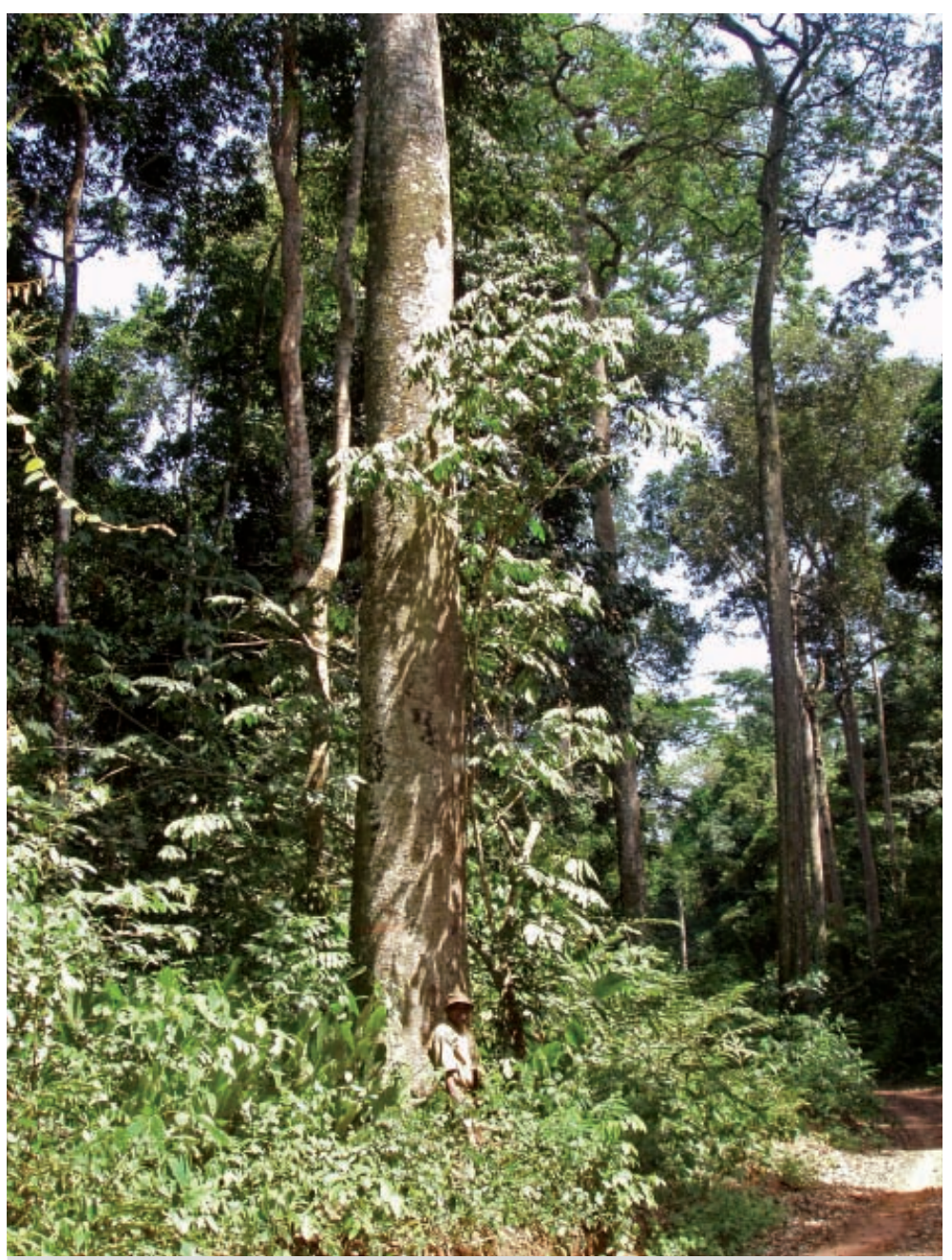

Celtis zenkeri en bord de route en République centrafricaine. Photo L. Durrieu de Madron.
Paramètres de l'exploitation forestière intervenant sur les stocks de carbone : revue des données existantes

Dans une forêt exploitée sélectivement, la quantité de carbone stockée est influencée par des facteurs et des processus internes au système : la composition en espèces, l'accroissement des arbres, la vitesse de décomposition des bois ou la richesse du sol. Ce stock est aussi influencé par des facteurs externes tels que le temps qui s'écoule entre deux coupes, l'intensité de prélèvement, les dommages de l'exploitation sur l'écosystème, la portion de volume de bois abattu laissée sur place. Pour mener cette étude, ce sont ces facteurs externes qui ont été retenus, car l'aménagiste et l'exploitant peuvent techniquement agir sur eux en fonction du contexte commercial et politique.

Les principales composantes du stock de carbone d'une forêt tropicale sont la biomasse aérienne, le bois mort, la litière, la matière organique du sol et les racines. La biomasse aérienne, qui est la composante majeure du stock de carbone, est également l'élément le plus affecté lors de la dégradation (GIBBs et al., 2007).

Il est important de faire une distinction entre court terme et moyen terme, car l'exploitation peut influencer la capacité de rétention de carbone d'un site et la vitesse de reconstitution et de séquestration du carbone (PINARD, CROPPER, 2000). À long terme, l'ouverture du couvert due aux trouées d'exploitation peut permettre une forte croissance du peuplement restant, permettant un stockage de carbone plus rapidement que pour une forêt non exploitée. L'impact négatif sur les stocks de carbone de l'exploitation à court terme peut se maintenir négatif, devenir nul ou positif, à plus ou moins long terme, en fonction des espèces qui se régénèrent (pionnières à faible densité de bois, ou espèces longévives à densité de bois élevée et de taille plus imposante) et de la capacité de résilience et de productivité du site. Cette capacité peut être altérée suite aux perturbations liées à l'exploitation telles que la compaction et l'érosion du sol qui suit le passage des engins en forêt (PINARD, CROPPER, 2000).

Pour cette étude, les estimations des gains de carbone ont été faites pour une durée de rotation de trente ans. Au cours d'une telle période, les opérations d'exploitation n'ont pas lieu en même temps, elles s'étalent tout au long de la durée de rotation. La date à laquelle une opération a lieu influence le bilan carbone en 30 ans. Celui-ci ne sera pas le même entre une assiette de coupe qui passerait en coupe au bout de 5 ans et une autre à 25 ans. La présente étude se borne à calculer le bilan carbone comme si toutes les opérations forestières avaient lieu simultanément et en considérant le bilan juste après ces opérations. Le devenir du carbone en forêt sur le long terme sort du cadre de l'étude. 


\section{Le phénomène de « repasse " dans l'exploitation conventionnelle}

Une concession forestière bénéficiant d'un plan d'aménagement se caractérise par la mise en exploitation d'un espace de forêt bien délimité et connu, l'assiette annuelle de coupe (Aac), différente chaque année, avec une marge légale de l'ordre de trois ans entre la première coupe et le dernier arbre abattu. L'étendue d'une concession définie comme série de production est ainsi exploitée entre deux passages en coupe au cours d'une rotation (terme utilisé dans les législations des pays du bassin du Congo). Dans le cas de concessions non aménagées (Ec), étant donné la connaissance incomplète des ressources exploitables, se déclenche le phénomène de repasse : l'exploitant intervient fréquemment au gré du marché du bois au sein de peuplements déjà parcourus en coupe.

L'impact comparé en termes de biomasse entre forêts, avec ou sans aménagement, n'a guère été étudié spécifiquement en Afrique centrale. Ce n'est pas le cas ailleurs; à titre d'exemple, une étude modélisant l'évolution des stocks de biomasse d'une forêt tropicale de Bornéo en Malaisie, gérée soit de manière durable (rotation de 40 ans), soit traditionnellement avec des repasses tous les 10 ans, montre une réduction importante de biomasse au cours du temps (Smith, Applegate, 2004).

Dans le cadre de l'étude, une simulation a été réalisée en utilisant des structures diamétriques issues de l'inventaire des forêts denses centrafricaines effectué par le Parn ${ }^{1}$ en 1994 , avec un calcul de reconstitution soit à 30 ans, soit chaque 10 ans, sans augmentation des Dme, sur un groupe de 14 essences couramment exploitées dans le bassin du Congo.

La méthode classique de calcul de taux de reconstitution (DURRIEU DE MADRon, Forni, 1997) a été utilisée dans les deux cas, avec des accroissements annuels diamétriques moyens de 0,3 à 1 centimètre par an ( $\mathrm{cm} / \mathrm{an})$ selon les essences, un taux de mortalité de $1 \%$ par an et $10 \%$ de dégâts d'exploitation. Les taux de reconstitution en effectifs sont de $39 \%$ en respectant une rotation de 30 ans et de $37 \%$ avec des repasses tous les 10 ans (respectivement 15 , 11 et $12 \%$ chaque décennie). La différence se situe essentiellement dans la répartition par classe de diamètre des arbres exploitables: ceux-ci ont le temps d'atteindre des plus grands diamètres en 30 ans sans repasse (figure 1).

Les prélèvements tous les 10 ans concernent moins d'arbres par hectare, entraînant des dégâts d'exploitation réduits sur le peuplement restant. La longueur des pistes de débardage par arbre augmente ; mais l'exploitant utilisera préférentiellement des pistes déjà existantes. Il est ainsi difficile d'estimer comparativement les dégâts sur les peuplements résiduels des deux types d'exploitation. Au vu de cette simulation africaine, la différence entre exploitation avec ou sans repasse peut être considérée comme négligeable en ce qui concerne l'impact sur la biomasse.

1 Projet d'aménagement des ressources naturelles, Poulin Thériault/Meccpt.

\section{Les séries de protection dans les concessions aménagées}

Dans le cas de concessions aménagées, différentes séries sont définies : de conservation, de protection, rurale/agroforestière/communautaire/agricole et de production. Les séries de protection et de conservation permettent de maintenir le stock de carbone initial, tandis que la série de production et la série rurale seront sujettes à une diminution du stock de carbone plus ou moins importante selon leur gestion. Dans les plans d'aménagement, ces séries de protection/conservation représentent en général de 2 à $10 \%$ de la superficie de la concession. Les cas suivants peuvent être cités (Ufa désigne une unité forestière d'aménagement) :

- 4 Ufa d'Alpicam au Cameroun : aucune zone de protection sur les trois premières Ufa et 3,8 \% sur la dernière, soit, en prenant la superficie utile de l'ensemble des $4 \mathrm{Ufa}$, 0,8\% mis en série de protection/recherche ;

- Ufa de Kabo (Cib) au Congo : série de conservation représentant $7,3 \%$ de la superficie de l'Ufa sans prendre en compte les zones inondées et les zones marécageuses ;

- Ufa de Lassio (Cora Wood) au Gabon : 0,5 \% (bureau d'études Sylvafrica, com. pers., qui fournit des valeurs de 2 et $3 \%$ pour deux autres permis au Gabon) ;

- 8 Ufa en République centrafricaine (projet Parpaf) fournissent des valeurs allant de 0 à $9 \%$ avec une moyenne de $4 \%$ (D. HUBERT, comm. pers.) ;

- 5 Ufa de Pallisco au Cameroun : la totalité des surfaces des séries de protection et de conservation correspond à 4,8\% de la superficie des concessions (D. HUBERT, comm. pers.).

En fait, les exploitants localisent ces séries de protection dans les zones les moins riches en bois exploitable et/ou les moins accessibles. Il est donc possible de considérer que ces séries de protection représentent nettement moins de $5 \%$ des zones qui auraient pu être exploitées sans plan d'aménagement. Des zones tampons peuvent également être délimitées le long des cours d'eau et des plans d'eau, ou des zones à valeur culturelle ou religieuse, ainsi que le long des zones à trop forte pente. Quoi qu'il en soit, ces mesures, en principe environnementales, ne sont guère utiles dans le cadre de la séquestration de carbone.

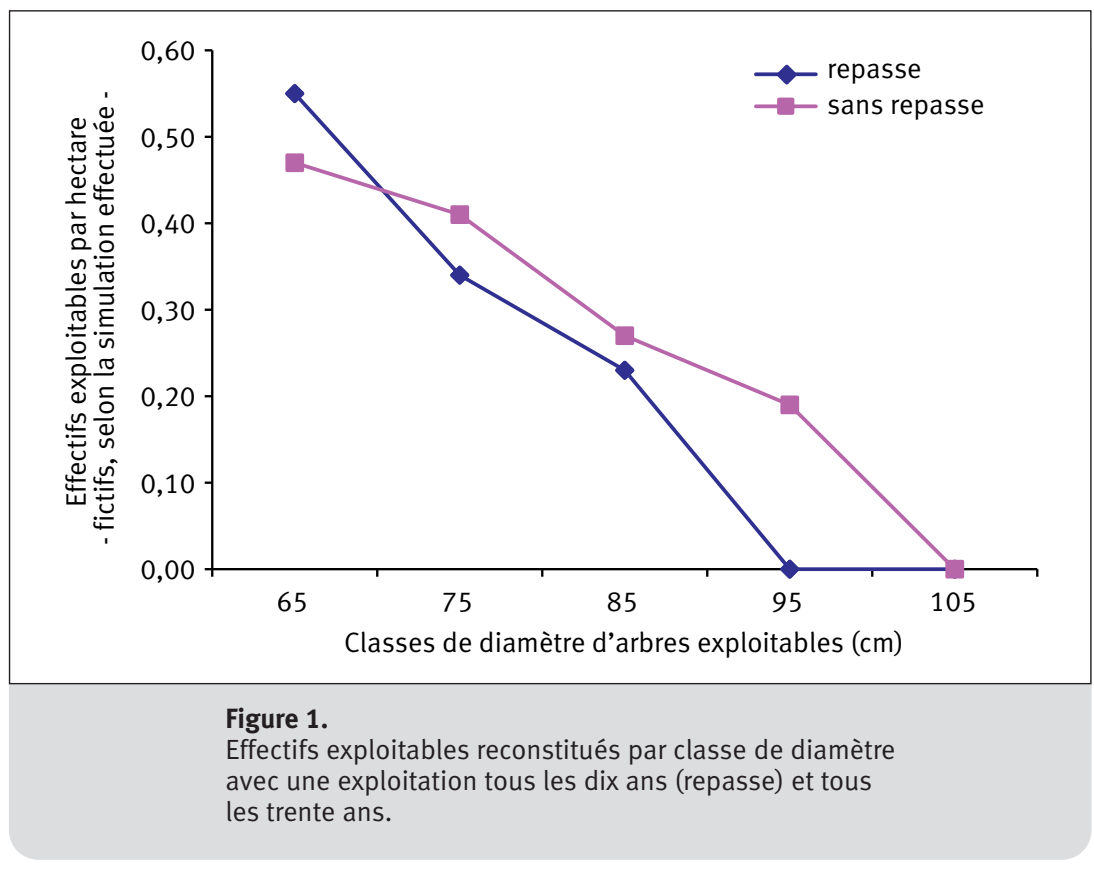




\section{Le choix du diamètre minimum des arbres exploitables et de la rotation}

Dans un plan d'aménagement d'un pays du bassin du Congo (République centrafricaine, Cameroun, Gabon, Congo, République démocratique du Congo), l'adéquation biologique des diamètres minimums d'exploitation (Dme) doit être vérifiée par le calcul d'un pourcentage de reconstitution des volumes exploitables qui conduit éventuellement à une augmentation des Dme en diamètres minimums " aménagés " (Dma), en fonction de la répartition du peuplement par classe de diamètre. La majorité des pays du bassin du Congo fixent différemment des seuils pour ce pourcentage de reconstitution, pour une essence seule ou pour un groupe d'essences (50\% au Cameroun pour un groupe de 20 essences «principales» ou $75 \%$ pour l'okoumé au Gabon). Quand la rotation n'est pas fixée directement par la législation (cas du Cameroun où la rotation est de 30 ans), son choix est fait suivant les Dma établis par espèce. L'augmentation des Dme maintient de fait un certain nombre de tiges hors du prélèvement.

Les volumes rendus ainsi inexploitables varient fortement :

- dans les 8 Ufa aménagées en République centrafricaine, les volumes rendus inexploitables parmi les arbres couramment exploités varient entre $13 \%$ et $40 \%$ des volumes initialement exploitables (supérieurs au Dme) (projet Parpaf, D. HUBERT, comm. pers.) ;

- dans l'Ufa 10.039 de Pallisco au Cameroun, 14,7 \% du volume des essences couramment exploitées a été rendu inexploitable (D. HUBERT, comm. pers.) ;

- dans l'Ufa de Lassio (Cora Wood) (L. Teillier, comm. pers.), au Gabon, $5 \%$ en volume et $8 \%$ en nombre de tiges sont rendus inexploitables sur 25 essences " objectifs";

- dans l'Ufa de Kabo (Cib) au Congo, 8,9\% des tiges des essences objectifs sont rendues inexploitables ;

- dans 4 Ufa d'Alpicam au Cameroun, 14 à $44 \%$ du volume des essences " aménagées » est rendu inexploitable avec une moyenne de $30 \%$ pour l'ensemble des 4 Ufa regroupées.

Les valeurs observées sont généralement supérieures à $10 \%$. Ces chiffres sont sans doute à pondérer, car le marché des bois rend aléatoire la commercialisation de certaines essences. Il est donc possible de considérer que la proportion des volumes réellement rendus inexploitables par un aménagement, comparé à ce qui serait exploité sans aménagement, est de l'ordre de $10 \%$. Quoi qu'il en soit, le choix pertinent des diamètres d'exploitation peut entraîner une économie nette de carbone.

\section{La valorisation des essences}

Les arbres de très grande taille sont parfois exclus de l'exploitation, par exemple les arbres de plus de deux mètres de diamètre (" arbres monuments » au Gabon). Mais cette loi n'est pas généralisée dans tous les pays du bassin du Congo. Le volume que représentent ces arbres par rapport au peuplement total peut être considéré comme négligeable du fait de leur rareté.
Un des principes de l'aménagement repose sur la valorisation des essences secondaires. Le développement industriel qui accompagne l'aménagement autorise la valorisation d'espèces auparavant dédaignées, mais acquérant de l'intérêt avec les nouvelles techniques de transformation et l'amélioration du transport. II en résulte une augmentation des prélèvements à l'hectare et donc une diminution du stock de carbone en forêt. Toutefois, cette valorisation des essences secondaires est difficile à évaluer faute de connaître ce qui se passerait sans aménagement en termes de volumes vendus.

\section{L'abattage et ses dégâts}

Parmi les techniques Efi (exploitation à faible impact), l'abattage directionnel est recommandé (JONKERS, VAN LEERSUM, 2000 ; JONKERS, 2000). L'avantage de cet abattage directionnel serait de positionner l'arbre de sorte qu'il n'y ait pas besoin de réaliser un débusquage et de passer directement au débardage, permettant ainsi de limiter les dégâts liés aux manœuvres du bulldozer ou du skidder. De plus, il est susceptible de faire tomber l'arbre dans une direction minimisant les dommages provoqués au sein du peuplement d'avenir resté sur pied. Lors d'un exercice mené en ce sens au Cameroun (DURRIEU DE MADRON et al., 1998), un écart moyen de $\pm 30^{\circ}$ a été constaté entre la direction désirée par l'abatteur et la direction réelle de chute de l'arbre abattu. En fait, cette imprécision sur la direction de chute réelle des arbres montre qu'il s'agit surtout d'abattage « contrôlé », qui consiste tout d'abord à faire tomber l'arbre de la manière la plus sûre pour l'équipe d'abattage, ensuite à augmenter l'efficience de l'utilisation de la tige exploitée (pas d'éclatement de la tige ni d'arrachage de fibre), puis à causer le moins de dégâts possible au peuplement d'avenir restant, et enfin à faciliter, autant que possible, l'extraction des billes (FAO, 2003).

La diminution des dommages par délianage préalable au passage en coupe n'est pas toujours effective en Afrique centrale. Par exemple, PARRen (1998), cité par JONKERS et VAN LeERSUM (2000), a constaté que ce délianage préalable ne réduisait pas la taille des ouvertures dans la canopée de la forêt étudiée au Sud-Cameroun. II n'est donc pas pris en compte dans cette étude.

\section{Impact carbone de l'abattage}

La quantité de carbone en jeu lors de l'abattage a été estimée dans l'étude de Brown et al. (2005) en République du Congo dans une concession sous plan d'aménagement pratiquant des techniques Efi, avec un diamètre moyen des arbres abattus de $123 \mathrm{~cm}$. Les auteurs estiment que la quantité de carbone extraite est de 6,82 tonnes de carbone (tC) par arbre abattu. Une quantité de 10,77 tC par trouée est perdue à court ou moyen terme du fait des dommages collatéraux sévères sur le peuplement avoisinant, et environ $6,74 \mathrm{tC}$ de résidus d'abattage $(49,7 \%$ de la biomasse totale de l'arbre abattu). Ces chiffres doivent être remis dans le contexte forestier dans lequel a eu lieu ce travail. L'exploitation dans d'autres zones peut ne rien avoir de commun avec le prélèvement ainsi décrit, notamment dans les zones déjà parcourues par l'exploitation. 


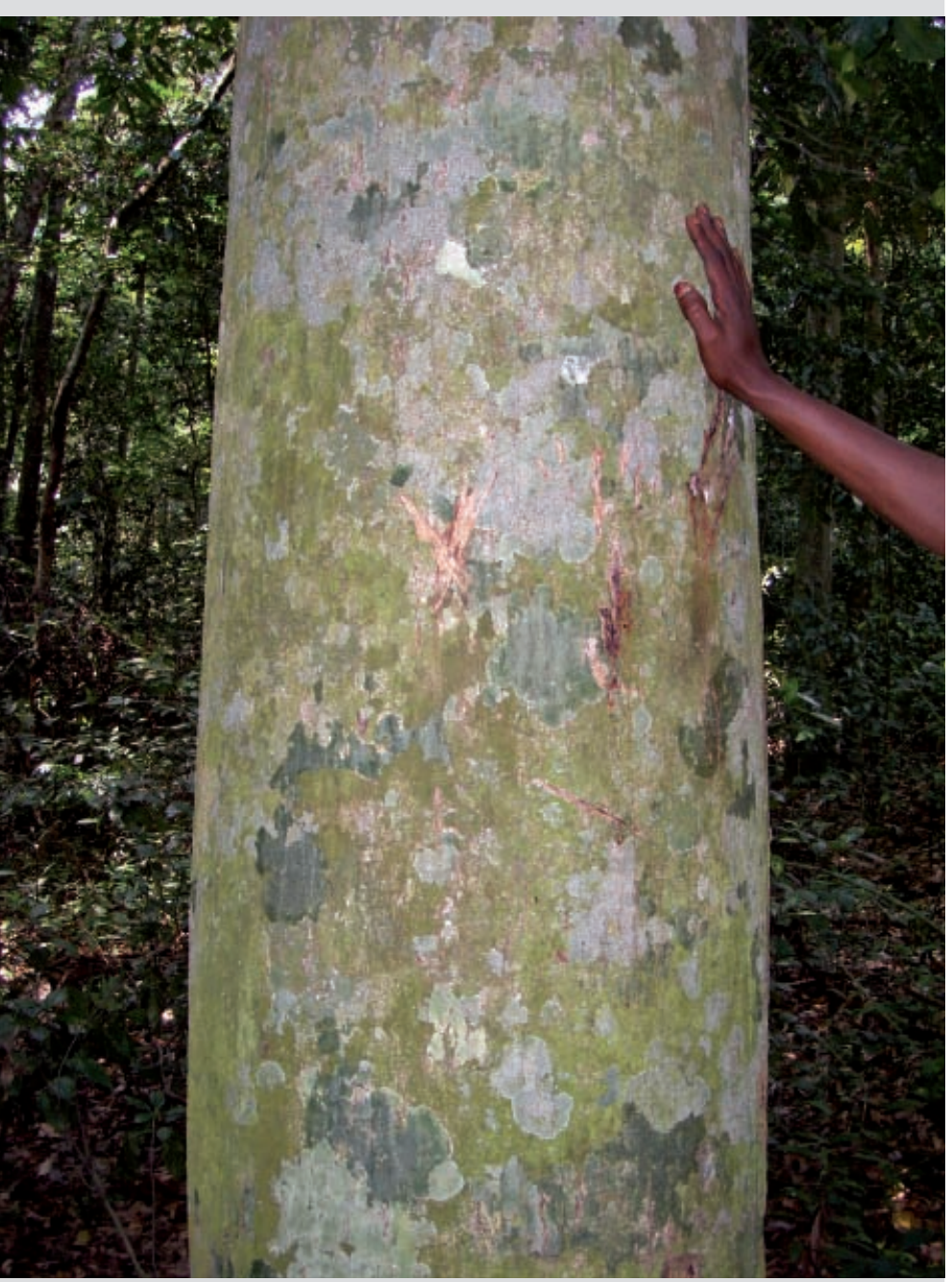

Wengue, Milettia laurentii, marqué sous diamètre minimum d'exploitation en République démocratique du Congo.

Photo L. Durrieu de Madron.

Au Gabon, une étude de recollement montre qu'il est possible d'obtenir une amélioration de $13 \%$ du coefficient de commercialisation (part du volume de fût abattu qui est réellement commercialisée) de l'okoumé en passant de l'abattage traditionnel à l'abattage contrôlé (WINGUE, 2002). Cette amélioration pourrait aller jusqu'à $20 \%$ selon DEmARQuez (1998). Cependant, l'amélioration des rendements d'abattage ne signifie pas la baisse des dégâts sur le peuplement restant. L'abattage directionnel peut diminuer de $25 \%$ les dommages sur les arbres avoisinants (JONKERS, VAN LEERSUM, 2000) s'il est effectivement appliqué, ce qui n'est pas le cas en Afrique centrale.

Le bilan final en carbone ne sera donc pas modifié par une amélioration des techniques d'abattage, seul le rendement matière sera augmenté.

\section{Les pistes de débardage}

Toute ouverture de piste de débardage occasionne l'ouverture d'une bande de forêt mais, contrairement à l'ouverture des routes, les plus grosses tiges sont épargnées. Les pertes de carbone sont principalement liées à la densité des pistes de débardage, aux dommages sur les arbres de faible diamètre, et aux dégâts occasionnés aux sols (compaction et érosion).
Au Cameroun, l'application de l'Efi par rapport à l'Ec, pour une intensité de prélèvement d'environ 1,5 arbre par hectare (ha), permet de diminuer de $13 \%$ la surface occupée par les pistes de débardage, ce qui correspond à une diminution de $11,3 \%$ de longueur de piste par arbre abattu (JONKERS, 2000). L'absence de balisage jusqu'aux tiges à exploiter entraîne une ouverture de 12 \% de longueur de piste inutile (МЕкок, 1995).

\section{Les routes principales, secondaires et les parcs à grumes}

Les routes principales et secondaires représenteraient globalement 1 à $2 \%$ de surface perturbée (DURRIEU DE MADRON et al., 1998). L'exploitation conventionnelle construit son infrastructure routière sans tenir compte de la richesse de ses peuplements et avec une cartographie peu précise. Certaines routes ne seront pas utilisées car non praticables par les grumiers (pentes trop fortes), tandis que d'autres nécessiteront un réseau important de bretelles et de débardage pour atteindre les essences à exploiter. Dans ces conditions, la perte de carbone est très importante par rapport à d'autres composantes telles que le débardage ou l'abattage. L'Efi permettrait d'éviter environ 30 \% des dommages liés à l'ouverture des routes et des parcs à grumes (JONKERS, VAN LEERSUM, 2000).

\section{Méthode d'estimation de l'impact global des différents modes d'exploitation}

\section{Le stock de carbone en forêt}

Le stock de carbone calculé par MugNIER et al. (2009) sur 4,8 millions d'hectares de forêt dans le bassin du Congo varie de 162 à 150 tonnes de carbone à l'hectare (tC/ha) en utilisant soit la méthode par défaut du GIEC (2006), soit la méthode allométrique (CHAVE et al., 2005). Ces calculs sont effectués sur 40900 ha de parcelles inventoriées et représentent une moyenne de plusieurs types de forêts y compris des forêts secondaires jeunes, donc non exploitables. Les stocks de carbone des différentes forêts varient de 83 à 227 tC/ha. En prenant une moyenne de $156 \mathrm{tC} /$ ha entre les deux méthodes, cela donne un stock de $118 \mathrm{tC} /$ ha épigé, en utilisant le ratio racines/biomasse épigée de 0,24 du GIEC (2006).

Le stock calculé par Brown et al. (2005) au Congo est de $276,7 \mathrm{tC} / \mathrm{ha} \pm 103,9$ (intervalle de confiance à $95 \%$ sur 10 échantillons de 0,13 ha dans la concession Cib). Retransformé en biomasse épigée avec le ratio 0,24 racines/biomasse épigée, il en résulte $223 \mathrm{tC}$ /ha épigées.

LEWIS et al. (2009), sur 163 ha (79 parcelles) répartis dans dix pays du bassin du Congo, fournissent un stock de $202 \mathrm{tC} /$ ha épigées, pour les arbres de plus de $10 \mathrm{~cm}$ de diamètre.

Djuikouo et al. (2010), sur 14 ha (14 parcelles) dans trois types de forêts différents (de la forêt sur terre ferme à la forêt inondable), donnent des valeurs ramenées en tC/ha épigées de 283 à 182 pour les arbres de plus de $10 \mathrm{~cm}$ de diamètre. 


\section{Les données utilisées}

Deux valeurs, qui encadrent celles de LewIS et al. (2009) et de Duuikouo et al. (2010), ont été retenues : celle de Mugnier et al. (2009) et celle de Brown et al. (2005). La valeur de MUGNIER et al. est la plus représentative du bassin du Congo et, pour des raisons de cohérence avec les paramètres utilisés pour les dégâts d'abattage, les pistes et les routes, est également utilisée la valeur de BRown et al. (2005), même si cette donnée s'avère ponctuelle.

De manière générale, les volumes de grumes extraits sont, pour 1 à 2 tiges exploitées par hectare, compris entre 10 et $40 \mathrm{~m}^{3} /$ ha en Afrique (SIST, 2000). Dans cette approche synthétique, sont retenus deux prélèvements types, l'un de $20 \mathrm{~m}^{3} /$ ha et l'autre de $10 \mathrm{~m}^{3} /$ ha.

L'étude a mis à profit les résultats de l'étude de Brown et al. (2005) au Nord de la République du Congo où, pour une intensité de prélèvement de 0,53 tige/ha (11 $\mathrm{m}^{3}$ /ha de volume de bois extrait), $8 \mathrm{tC} /$ ha sont "libérées » par l'exploitation forestière de type «Espa + Efi », donnant un chiffre de 10,2 tC/ha par extrapolation sur 1194 ha par image satellite. Un mètre cube extrait représente 0,27 tC extraite de la forêt et $0,46 \mathrm{tC}$ de dégâts sur le peuplement résiduel et les résidus d'exploitation, soit $0,73 \mathrm{tC} / \mathrm{m}^{3}$. Cette valeur constitue le facteur de conversion biomasse.

Un mètre de piste de débardage de largeur moyenne de 4,5 m libère 6,83 kilogrammes de carbone $(\mathrm{kgC})$ et un mètre carré de route libère 0,028 tC (BRown et al., 2005).

\section{Méthodes de calcul des impacts en carbone}

Les impacts en carbone des paramètres sont calculés comme suit :

- Série de protection : superficie de la série de protection multipliée par le tonnage de biomasse qui aurait été extrait par hectare de la forêt (volume moyen extrait par hectare multiplié par le facteur de conversion biomasse comprenant les dégâts) ;

$\mathrm{I}_{\mathrm{Sp}}=\mathrm{S} \times\left(\mathrm{V}_{\mathrm{m}} \times \mathrm{F}_{\mathrm{c}}\right)$

Avec $I_{S p}$, l'impact en carbone sur la série de protection (tC) ; $\mathrm{S}$, la superficie de la série de protection (ha) ;

$\mathrm{V}_{\mathrm{m}}$, le volume moyen extrait par hectare $\left(\mathrm{m}^{3} / \mathrm{ha}\right)$;

$\mathrm{F}_{c}$, le facteur de conversion biomasse comprenant les dégâts $\left(\mathrm{tC} / \mathrm{m}^{3}\right)$.

- Augmentation des Dme : volume moyen extrait par hectare multiplié par le pourcentage du volume rendu inexploitable par l'augmentation des DME, multiplié par le facteur de conversion biomasse comprenant les dégâts multiplié par la superficie productive ;

$\mathrm{I}_{\text {Dme }}=\mathrm{V}_{\mathrm{m}} \times \mathrm{V}_{0} \times \mathrm{F}_{\mathrm{c}} \times \mathrm{S}_{\text {prod }}$

Avec $I_{\text {Dme }}$, l'impact en carbone avec l'augmentation des Dme (tC);

$V_{m}$, le volume moyen extrait par hectare $\left(\mathrm{m}^{3} / \mathrm{ha}\right)$;

$\mathrm{V}_{0}$, pourcentage du volume rendu inexploitable par l'augmentation des DME (\%) ;

$\mathrm{F}_{c}$, le facteur de conversion biomasse comprenant les dégâts (en $\mathrm{tC} / \mathrm{m}^{3}$ ) ;

$\mathrm{S}_{\text {prod}}$, la superficie productive (ha).
- Piste de débardage : tonnage de carbone «libéré » par mètre linéaire de piste ouverte, en comptant $200 \mathrm{~m}$ de piste par arbre et un arbre/ha exploité multiplié par le pourcentage de piste économisé ;

$\mathrm{I}_{\text {Pdeb }}=\mathrm{N}_{\text {arb }} \times \mathrm{T}_{\text {piste }} \times \mathrm{L}_{\text {piste }} / 200$

Avec, I Pdeb l'impact des pistes de débardage (tC) ;

$\mathrm{N}_{\text {arb }}$, le nombre d'arbres exploités par hectare (arbres/ha) ;

$\mathrm{T}_{\text {piste }}$, le taux de piste économisée (\%);

$\mathrm{L}_{\text {piste}}$, la longueur de piste ouverte $(\mathrm{m})$.

- Routes : tonnage de carbone «libéré » par mètre carré de route ouverte multiplié par le pourcentage de superficie occupé par les routes dans la concession multiplié par le pourcentage d'économie de dégâts ;

$\mathrm{I}_{\text {route }}=\mathrm{Q}_{\text {carb }} \times \mathrm{S}_{\text {route }} \times \mathrm{T}_{\text {r./con. }} \times \mathrm{T}_{\text {dégats } 0}$

Avec $I_{\text {route }}$, l'impact des routes (tC);

$\mathrm{Q}_{\text {carb }}$, la quantité de carbone « libérée » $(\mathrm{t})$;

$\mathrm{T}_{\mathrm{r} . / \text { con. }}$, le taux de superficie occupée par les routes dans la concession (\%) ;

$\mathrm{T}_{\text {dégatso }}$, le taux d’économie de dégâts (\%).

\section{Résultats : l'impact global des modes d'exploitation}

Les données ont été calculées pour un permis hypothétique de 200000 ha avec $10 \%$ de série agricole et $3 \%$ de série de protection/conservation, soit 174000 ha de série de production. Les résultats du calcul de l'impact carbone de chaque opération forestière, avec un plan d'aménagement et avec l'utilisation de techniques à faible impact, sont présentés dans le tableau I. Ces valeurs jouent un rôle d'exemple et restent théoriques.

L'exploitation conventionnelle sur 180000 ha libérerait selon les deux taux d'extraction :

- pour une exploitation moyenne de $10 \mathrm{~m}^{3} / \mathrm{ha}(0,5$ arbre exploité/ha) : $10 \mathrm{~m}^{3} \times 0,73 \mathrm{tC} \times 180000=1314000$ tC, auxquels il faut ajouter les pistes ( $7 \%$ de la superficie productive $\times 0,00683 \mathrm{kgC} / \mathrm{m}$ ), les routes ( $1 \%$ de la superficie productive $\times 0,028 \mathrm{kgC} / \mathrm{m}^{2}$ ), respectivement $245800 \mathrm{tC}$ et 504000 tC, soit un total de 1941000 tC;

- pour une exploitation moyenne de $20 \mathrm{~m}^{3} / \mathrm{ha}$ (1 arbre exploité/ha) : $20 \mathrm{~m}^{3} \times 0,73 \mathrm{tC} \times 180000=2628000$ tC, auxquels il faut ajouter les pistes et les routes, respectivement 245800 tC et 504000 tC, soit un total de 3378000 tC.

La mise sous aménagement entraînerait une amélioration théorique, avec les hypothèses posées ci-dessus (protection, augmentation des Dme...), de 171000 tC pour une exploitation de $10 \mathrm{~m}^{3} /$ ha et de $342000 \mathrm{tC}$ pour une exploitation de $20 \mathrm{~m}^{3} / \mathrm{ha}$, soit une diminution de 9 à $10 \%$ des pertes en carbone de la forêt par rapport à l'exploitation conventionnelle (sans prendre en compte la décomposition in situ des bois morts, etc.). L'essentiel de l'économie en carbone provient de l'augmentation des diamètres minimums d'exploitation. La compensation par les séries de protection n'induit pas une réelle diminution des dégâts d'exploitation mais une mise hors exploitation d'une partie de la forêt. 
Les gains qui pourraient être induits par la pratique d'une exploitation à faible impact seraient ceux des pistes et des routes, soit, pour une exploitation de $10 \mathrm{~m}^{3} / \mathrm{ha}, 161000 \mathrm{tC}$ et, pour une exploitation de $20 \mathrm{~m}^{3} / \mathrm{ha}, 175500 \mathrm{tC}$, soit 5 à $8 \%$ des pertes en carbone dues à l'exploitation conventionnelle. Dans ce cas, est effectivement induite une réduction des dégâts sur une zone d'exploitation donnée.

La gestion d'une forêt correctement aménagée, mettant à profit les techniques d'exploitation à faible impact, entraînerait, pour une rotation de trente ans, une nette amélioration du carbone déstocké. Sa réduction serait de l'ordre du sixième par rapport à une gestion conventionnelle.

\section{Discussion}

\section{Ordre de grandeur et fiabilité des résultats}

Les résultats présentés permettent de donner un ordre de grandeur des différences possibles, en termes de carbone, entre l'exploitation conventionnelle, l'exploitation sous plan d'aménagement et l'exploitation à impact réduit. Pour obtenir des estimations plus rigoureuses, il faudrait mener d'autres études avec des intensités d'exploitation variables, au sein de différentes structures de peuplement.

\section{Erreur sur l'évaluation de la biomasse}

En outre, il faut comparer ces chiffres avec l'incertitude sur les calculs de biomasse (facteur d'estimation du houppier, ratio de racines sur biomasse épigée, erreurs dues aux équations allométriques...).

BRown et al. (2005) fournissent une erreur dans l'estimation de la biomasse de la forêt de $37 \%(276,7 \pm 103,9$ tC/ha $)$ sur une surface réellement échantillonnée très restreinte. De plus, une erreur sur le carbone libéré par l'exploitation forestière est estimée à 9,8 \%, après extrapolation par photo aérienne, sur 1194 ha.

Pour l'étude à très grande échelle de MUGNIER et al. (2009), il n'y a pas d'erreur fournie, mais la simple différence entre 150 et $162 \mathrm{tC} / \mathrm{ha}$, calculées par la méthode de CHAVE et al. (2005) et la méthode du GIEC (2006), représente déjà une incertitude de 9,2 \%. Pour la mise au point des modèles sur 27 sites pantropicaux (CHAVE et al., 2005), une erreur standard de $15 \%$ est avancée, entre la biomasse mesurée des différents sites et celle prévue par les équations générales. En outre, aucun de ces échantillons ne provient de forêts africaines.

Les deux méthodes, facteur d'estimation du houppier (Bef) et équation allométrique, reposent sur des échantillons pantropicaux non africains (BRown et al., 1989 ; Brown, Lugo, 1992 ; ChAVE et al., 2005). Le Bef par défaut

\section{Tableau I. \\ Estimation des impacts carbone des différentes opérations d'exploitation/aménagement d'une concession hypothétique de 200000 ha, entraînés par la mise sous aménagement (Espa) puis par l'utilisation de techniques d'exploitation à faible impact (Espa + Efi), par rapport à une exploitation conventionnelle (Ec).}

Actions techniques

Repasse par rapport au respect des rotations

Séries de protection

Augmentation des diamètres minimums d'exploitation

Valorisation des essences secondaires Interdiction d'abattage des très gros arbres Abattage amélioré

Délianage

Pistes

Parcs à grumes

Routes
Forêt aménagée (Espa)

Gain non prouvé

Moins de $5 \%$ en surface

Estimé à $10 \%$ du volume des essences couramment exploitées

Augmentation du nombre de tiges exploitées à l'hectare pouvant entraîner un déstockage de carbone difficile à estimer

Gain minime en carbone car ces arbres sont rares et la règle n'existe pas dans tous les pays
Forêt aménagée avec exploitation

à faible impact (Espa + Efi)
Impact carbone par rapport à l'exploitation conventionnelle (Ec) 


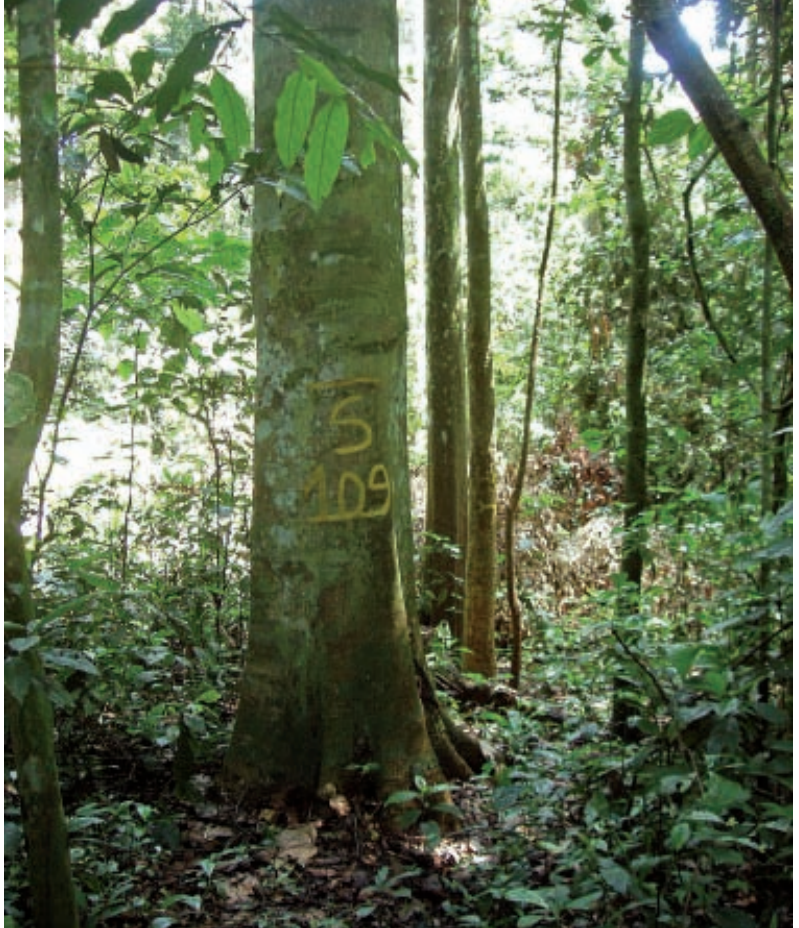

supérieure 190 t/ha. Une mesure récente au Cameroun (DURRIEU DE MADRon, données non publiées) sur 55 arbres fournit une valeur de 1,63 avec une très forte disparité des valeurs (écart-type de 1,85). La simple utilisation du Bef de 1,74 ou de 1,63 entraîne une différence de 9,3\%.

Dans des forêts secondaires à Sumatra, KetTering et al. (2001) montrent la possibilité d'avoir une erreur globale de 36 à 51 \% sur l'estimation de la biomasse en utilisant des densités spécifiques de bois par défaut.

Murali et al. (2005), utilisant les données de 95 sites tropicaux publiées dans la littérature, ont développé des équations pour estimer la biomasse à partir de la surface terrière, la densité et la hauteur des arbres dans des forêts naturelles. Les erreurs calculées en forêt sempervirente varient entre 60 et $88 \%$.

L'étude réalisée en Guyane sur un hectare de forêt mesurés et pesés après dessiccation - a permis de déterminer une relation allométrique entre le diamètre et la biomasse aérienne. L'erreur entre l'estimation de la biomasse par cette équation et la valeur issue de la pesée est de 6,8\%. Une estimation en utilisant la médiane des classes de diamètre entraîne une erreur de 5 \% par rapport à la valeur issue de la pesée (LESCURE et al., 1983). Cette technique par destruction du couvert et pesée représente la technique d'évaluation de biomasse la plus proche de la réalité. Il est certes illusoire de chiffrer l'erreur globale mais, d'après les travaux de MURALI et al. (2005) et ceux de Kettering et al. (2001), elle risque d'être largement supérieure à cette valeur minimale de 7 \%.

\section{Comparaison avec les valeurs dues à la mise sous aménagement et l'utilisation de techniques d'exploitation à faible impact}

L'estimation du gain de carbone d'environ $10 \%$ qu'induit la mise en aménagement par rapport à une exploitation conventionnelle est ainsi comparable à l'erreur minimale sur l'estimation de la biomasse, ainsi qu'à l'erreur calculée par extrapolation par BRown et al., ainsi que celle issue de la différence entre les deux méthodes de calcul utilisées par MUGNIER et al.

Les gains en carbone obtenus grâce à l'utilisation de techniques d'exploitation à faible impact sont de l'ordre de 5 à $8 \%$ au sein d'une forêt aménagée. Ils sont inférieurs à l'erreur minimale sur l'estimation de la biomasse et les erreurs de Brown et al. (2005) et MugNIER et al. (2009) et devraient être largement inférieur à l'incertitude globale sur l'estimation de la biomasse.

L'interprétation de ces résultats ne représente, dans l'état actuel des connaissances, qu'une première approche à confirmer. De plus, comme le montrent les différentes valeurs de stock de carbone des différents types de forêts citées par MUGNIER et al. (2009), les différents paramètres utilisés pour évaluer l'effet de la mise sous aménagement et de l'utilisation des techniques d'exploitation à faible impact ne sont que des études ponctuelles, sujettes à de fortes variations régionales.
Tali, Erytrophleum guineense, conservé sur pied en tant que semencier en vue d'assurer la régénération. Photo L. Durrieu de Madron.

\section{Comparaison avec l'Amérique du Sud}

Au Brésil, dans l'État du Pará, pour une extraction de volume de bois de $30 \mathrm{~m}^{3} / \mathrm{ha}$, l'exploitation à faible impact diminue d'environ $5 \%(\approx 9,4 \mathrm{tC} / \mathrm{ha}$ ) le stock de carbone de la forêt (186 tC/ha) au bout de 30 ans (KeLLER et al., 2004). L'exploitation conventionnelle a également été étudiée, montrant un impact de $10 \%$ sur le stock de biomasse initial de la forêt. Mais elle ne prend pas en considération les facteurs tels que les repasses, les routes et le coefficient de commercialisation. L'écart entre les deux modes d'exploitation serait donc plus important qu'annoncé dans cette étude. Or, Putz et al. (2008) se fondent sur cette étude pour extrapoler l'impact de l'amélioration des méthodes d'exploitation pour l'Afrique centrale. D'une part, il y aurait un potentiel d'amélioration plus important que ceux qu'ils annoncent ; mais d'autre part, en ce qui concerne l'Afrique centrale, pour un même mode d'exploitation, les dommages liés à l'exploitation peuvent être bien moindres qu'en Amérique du Sud pour extraire un même volume. II s'avère que les structures forestières ne sont pas les mêmes entre les deux continents, les arbres exploités en Amérique du Sud étant généralement moins volumineux que ceux rencontrés en Afrique centrale. De plus, le nombre de tiges prélevées est plus important en Amazonie centrale, quatre à cinq tiges à l'hectare $\left(40-70 \mathrm{~m}^{3} / \mathrm{ha}\right)$ contre une à deux tiges (10-40 m³/ha) en Afrique (SIST, 2000).

Les dommages en termes de biomasse pour une quantité de biomasse extraite donnée sont, par exemple, de $60 \%$ moins importants dans le cas de l'étude en République du Congo par rapport à une étude menée en Bolivie dans les mêmes conditions (BRown et al., 2005).

En ce qui concerne la reconstitution, MAzzEl et al. (2010), sur 172 ha de forêt de terre ferme dans l'État du Pará au Brésil, exploités avec les techniques à faible impact, préconisent de réduire de moitié l'intensité des prélèvements actuels (six arbres par hectare) pour pouvoir récupérer la biomasse épigée en trente ans. 


\section{Conclusion}

La gestion forestière durable en Afrique centrale peut donc avoir un impact favorable et significatif sur les stocks de carbone à l'échelle d'une concession.

En tenant compte des hypothèses retenues dans cette étude, la gestion durable d'une concession forestière entraînerait une amélioration théorique d'environ $10 \%$ des pertes en carbone de la forêt pour des prélèvements de bois de 10 à $20 \mathrm{~m}^{3} / \mathrm{ha}$; et cela par rapport à l'exploitation conventionnelle sans gestion prévisionnelle. L'essentiel de l'économie en carbone provient de la mise en place des séries de protection et surtout de l'augmentation des diamètres minimums d'exploitation.

La diminution des pertes de carbone qui pourrait être induite par la pratique d'une exploitation à faible impact serait celle des pistes, soit, pour une exploitation de 10 à $20 \mathrm{~m}^{3} / \mathrm{ha}$, 5 à $8 \%$ des pertes en carbone dues à l'exploitation conventionnelle.

La gestion raisonnable et l'utilisation de techniques forestières appropriées peuvent ainsi induire un gain cumulé en carbone de l'ordre de 15 à $18 \%$. Par contre, il est pour l'instant impossible de prouver qu'une mise sous aménagement d'une concession ou l'utilisation de techniques d'exploitation à faible impact entraîne un impact significativement mesurable sur le stockage de carbone de la forêt, étant donné l'incertitude globale sur l'estimation de la biomasse avec les techniques actuelles.

Cependant, une exploitation sous plan d'aménagement peut amener une baisse des stocks de biomasse à travers plusieurs cycles de rotation si l'intensité de prélèvement s'avère excessive. Il est essentiel de définir un seuil d'intensité de prélèvement ou seuil de résilience de la forêt, de sorte que l'exploitation soit en accord avec la définition de la gestion forestière durable.

Il n'existe pas actuellement d'étude de terrain en Afrique centrale qui compare les modes d'exploitation; les travaux de recherche exigeraient de très grandes superficies d'étude avec des répétitions de parcelles et de traitements pour un même type d'exploitation. Les quelques dispositifs de recherche récemment mis en place dans la région vont, certes, fournir des données intéressantes ; mais il serait aussi nécessaire de développer les études ponctuelles pour déterminer les améliorations amenées en termes d'exploitation à faible impact (routes, pistes, abattage amélioré) dont les données disponibles sont trop rares. De même, un réseau de placettes permanentes permettrait de bien caractériser l'évolution des peuplements après exploitation et donc le bilan à long terme de la biomasse et du carbone.

\section{Remerciements}

Les auteurs remercient vivement les sociétés qui ont accepté de fournir leurs données : Alpicam, Pallisco, Cora Wood, Cib, le bureau Sylvafrica et le projet Parpaf.

\section{Références bibliographiques}

ASNER G., 2006. Measuring carbon emissions from tropical deforestation: an overview. New York, États-Unis, Environmental Defense Fund, $11 \mathrm{p}$.

BROWN S., 1997. Estimating biomass and biomass change of tropical forests: a primer. Rome, Italie, Fao, Forestry Paper, 134, $55 \mathrm{p}$.

BROWN S., GILLEPSIE A. J. R., LUGO A., 1989. Biomass estimation methods for tropical forests with applications to forest inventory data. Forest Science, 38: 881-902.

BROWN S., LUGO A. E., 1992. Above ground biomass estimates for tropical moist forests of the Brazilian Amazon. Interciencia, 17: 8-18.

BROWN S., PEARSON T., MOORE N., PARVEEN A., AMBAGIS S., SHOCH D., 2005. Impact of selective logging on the carbon stocks of tropical forests: Republic of Congo as a case of study. Deliverable 6: logging impacts on carbon stocks. Little Rock, AR, États-Unis, Winrock International, 21 p.

CHAVE J., ANDALO C., BROWN S., CAIRNS M. A., CHAMBERS J. Q., EAMUS D., FÖLSTER H., FROMARD F., HIGUCHI N., KIRA T., LESCURE J.-P., NELSON B. W., OGAWA H., PUIG H., RIERA B., YAMAKURA T., 2005. Tree allometry and improved estimation of carbon stocks and balance in tropical forests. Ecologia, 145: 87-99.

DEMARQUEZ B., 1998. L'aménagement durable vécu par un exploitant forestier. Canopée, 11 : 22-23.

DENMAN K. L., BRASSEUR G., 2007. Couplings between changes in the climate system and biogeochemistry: the physical science basis. Contribution of working group I to the fourth assessment report of the intergovernmental panel on climate change. In: Solomon S., Qin D., Manning M., Chen Z., Marquis M., Averyt K. B., Tignor M., Miller H. L. (eds). Cambridge, Royaume-Uni, Cambridge University Press, p. 489-588.

DJUIKOUO M. N. K., DOUCET J.-L., NGUEMBOU C. K., LEWIS, S. L., SONKÉ B., 2010. Diversity and aboveground biomass in three tropical forest types in the Dja Biosphere Reserve, Cameroon. African Journal of Ecology, 48 (4): 1053-1063.

DURRIEU DE MADRON L., FORNI E., 1997. Aménagement forestier dans l'Est du Cameroun : structure du peuplement et périodicité d'exploitation. Bois et Forêts des Tropiques, 254 : 39-64.

DURRIEU DE MADRON L., FORNI E., MEKOK M., 1998. Les techniques d'exploitation à faible impact en forêt dense humide camerounaise. Montpellier, France, Cirad-Forêt, série Forafri, $\mathrm{n}^{\circ} 17,28 \mathrm{p}$.

FAO, 2003. Code régional d'exploitation forestière à faible impact dans les forêts denses tropicales humides d'Afrique centrale et de l'Ouest. Rome, Italie, Fao, Forestry Paper, 134, $131 \mathrm{p}$. 
GIBBS H. K., BROWN S., O’NILES J., FOLEY J., 2007. Monitoring and estimating tropical forest carbon stocks: making REDD a reality. Environmental Research Letters, 2, 14 p. doi:10.1088/1748-9326/2/4/045023.

GIEC, 2006. Lignes directrices 2006 du Giec pour les inventaires nationaux de gaz à effet de serre. Vol. 4 : Agriculture, foresterie et autres affectations des terres. Kanagawa, Japon, Institute for Global Environnemental Strategies, vol. 4 : 4.46-4.52.

JONKERS W., 2000. Logging, damage and efficiency: a study on the feasibility of reduced impact logging in Cameroon. Final report. Kribi, Cameroun, The Tropenbos-Cameroon Programme, $57 \mathrm{p}$.

JONKERS W., VAN LEERSUM G., 2000. Logging in south Cameroon: current methods and opportunities for improvement. International Forestry Review, 2 (1): 11-16.

KELLER M., ASNER G., SILVA N., PALACE M., 2004. Sustainability of selective logging of upland forests in the Brazilian Amazon: carbon budgets and remote sensing as tools for evaluating logging effects. In: Zarin D., Alavalapati J., Putz F., Schmink M. (eds). Working forests in the Neotropics. New York, États-Unis, Columbia University Press, p. 41-63.

KETTERING Q., COE R., VAN NOORDWIJK M., AMBAGAU Y., PALM C., 2001. Reducing uncertainty in the use of allometric biomass equations for predicting above ground biomass in mixed secondary forests. Forest Ecology and Management, 146 (1-3): 199-209.

LAPORTE N. T., STABACH J. A., GROSCH R., LIN T. S., GOETZ S. J., 2007. Expansion of industrial logging in Central Africa. Science, 316 (5830): 1451.

LESCURE J. P., PUIG H., RIERA B., LECLERC D., BEEKMAN F., BENETEAU A., 1983. La phytomasse épigée d'une forêt dense en Guyane française. Acta Ecologica, Ecologia Generalis, 4 (3) : 237-251.

LEWIS S., LOPEZ-GONZALEZ G., SONKÉ B., AFFUM-BAFFOE K., BAKER T., OJO L., PHILLIPS O., REITSMA J., WHITE L., COMISKEY J., DJUIKOUO M. N., EWANGO C., FELDPAUSCH T., HAMILTON A., GLOOR M., HART T., HLADIK A., JON LLOYD LOVETT J., MAKANA J. R., MALHI Y.. MBAGO F., NDANGALASI H., PEACOCK J., PEH K., SHEIL D., SUNDERLAND T., SWAINE M., TAPLIN J., TAYLOR D., THOMAS S., VOTERE R., WÖLL H., 2009. Increasing carbon storage in intact African tropical forests. Nature, 457: 1003-1006.

MAZZEI L., SIST P., RUSCHEL A., PUTZ F., MARCO P., PENA W., RIBEIRO FEREIRA J. E., 2010. Above ground biomass dynamics after reduced impact logging in the eastern Amazon. Forest Ecology and Management, 259 (3): 367-373.

MEKOK M., 1995. Incidence de la planification sur la pratique actuelle de l'exploitation. Cirad, Rapport technique Aménagement pilote intégré Dimako, Cameroun, 12 p.
MUGNIER A., CASSAGNE B., BAYOL N., LAFON C., 2009. Estimation des stocks de carbone des forêts du bassin du Congo pour le REDD : étude comparative conduite sur 22 types forestiers, 4 pays et un dispositif d'aménagement 4,8 millions d'ha. XIII congrès forestier mondial, Buenos Aires, Argentine, 18-23 octobre 2009.

MURALI K. S., BHAT D. S., RAVINDRANATH N. H., 2005. Biomass estimation equations for tropical deciduous and evergreen forests. International Journal of Agricultural Resources, Governance and Ecology, 4 (1): 81-92.

PINARD M., CROPPER W., 2000. Simulated effects of logging on carbon storage in dipterocarp forest. Journal of Applied Ecology, 37: 267-283.

PUTZ F. E., ZUIDEMA P. A., PINARD M. A., BOOT R. G. A., SAYER J. A., SHEIL D., SIST P., ELIAS VANCLAY J. K., 2008. Improved tropical forest management for carbon retention. PLoS Biology, 6: 12.

SIST P., 2000. Les techniques d'exploitation à faible impact. Bois et Forêts des Tropiques, 265 : 31-43.

SMITH J., APPLEGATE G., 2004. Could payments for forest carbon contribute to improved tropical forest management? Forest Policy and Economics, 6: 153-167.

WINGUE T. R., 2002. Étude de recollement : évaluation du volume commercial et pertes de bois à la société de bois de Lastourville (S. B. L.), Gabon. Mémoire de fin d'études, Enef, Libreville, Gabon. 